\title{
Les Tchouvaches et les peuples finno-ougriens : une expérience de contacts interculturels dans la région Volga-Oural
}

The Chuvash and the Finno-Ugric Peoples: An Experience of Intercultural Contacts in the Urals and Volga Region

ЧУВАШИ И ФИННО-УГОРСКИЕ НАРОДЫ: ОПЫТ МЕЖКУЛЬТУРНЫХ КОНТАКТОВ В УРАЛО-ПОВОЛЖЬЕ

\section{Ekaterina Jagafova}

Traducteur : Eva Toulouze

\section{(2) OpenEdition}

1 Journals

Édition électronique

URL : https://journals.openedition.org/efo/7117

DOI : $10.4000 /$ efo. 7117

ISSN : 2275-1947

Éditeur

INALCO

Édition imprimée

ISBN : 978-2-343-12463-6

ISSN : 0071-2051

Référence électronique

Ekaterina Jagafova, «Les Tchouvaches et les peuples finno-ougriens : une expérience de contacts interculturels dans la région Volga-Oural », Études finno-ougriennes [En ligne], 48 | 2017, mis en ligne le 19 juillet 2017, consulté le 08 juillet 2021. URL : http://journals.openedition.org/efo/7117 ; DOI : https://doi.org/10.4000/efo.7117

Ce document a été généré automatiquement le 8 juillet 2021.

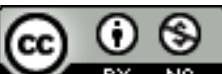

Études finno-ougriennes est mis à disposition selon les termes de la Licence Creative Commons Attribution - Pas d'Utilisation Commerciale 4.0 International. 


\section{Les Tchouvaches et les peuples finno-ougriens : une expérience de contacts interculturels dans la région Volga-Oural}

The Chuvash and the Finno-Ugric Peoples: An Experience of Intercultural

Contacts in the Urals and Volga Region

ЧУВАШИ И ФИННО-УГОРСКИЕ НАРОДЫ: ОПЫТ МЕЖКУЛЬТУРНЫХ КОНТАКТОВ В

УРАЛО-ПОВОЛЖЬЕ

Ekaterina Jagafova

Traduction : Eva Toulouze

\section{NOTE DE L'AUTEUR}

Cette recherche a été menée dans le cadre du projet RNGF 14-01-00360 « La culture de la fête chez les Tchouvaches au tournant des $\mathrm{Xx}^{\mathrm{e}}$-XxI ${ }^{\mathrm{e}}$ siècles ".

\section{Introduction}

1 Dans la recherche portant sur les peuples de la région Oural-Volga, une grande attention a été portée ces dernières décennies au problème de l'interaction interethnique (Korostelev, 2007, 2009, 2010 ; Iagafova, 2010; Popova, 2010 ; Sadikov, 2010; Danilko et al., 2010): c'est dans ce cadre que l'on étudie le caractère de l'interaction et de l'adaptation de groupes avoisinants, les facteurs et les situations des contacts interethniques, et que l'on identifie empiriquement les frontières ethniques qui passent entre eux. Cette approche repose sur une interprétation de l'ethnicité en tant que forme d'organisation sociale de la différence culturelle (Barth, 2006, p. 16). 
2 Les frontières ethniques passent par des marques de distinction, des dichotomies ethniques (langue, type d'habitation, habillement, mode de vie, etc.), qui, en cas d'interaction, tendent à varier, aboutissant à la formation de codes et de valeurs semblables entre les groupes en contact. Le niveau de ressemblance ou de différence entre les valeurs des différents groupes détermine aussi le caractère de l'interaction interethnique, par exemple par l'intermédiaire d'interdits de groupes mixtes, de solidarité interne aux groupes (Barth, 2006, p. 16-18, 21). Il est cependant important pour une bonne coexistence de l'ensemble social en tant que système polyethnique que les différences se complètent et ne soient pas entre elles en opposition.

3 Le caractère de l'adaptation mutuelle des groupes ethniques est influencé par toute une série de facteurs: écologiques, démographiques, et par conséquent des paramètres économiques (liés à la production), sociaux et politiques (Barth, 2006, p. 22-41). L'importance de chacun d'entre eux est corrélée à la situation concrète d'interaction dont dépendent les effets des contacts interethniques, dans les sphères les plus diverses : habitat, production, mariages, pratiques rituelles et festives, etc.

Ces régularités sont importantes d'un point de vue méthodologique pour l'étude des contacts des Tchouvaches avec leurs voisins finno-ougriens: Maris, Oudmourtes, Mordves.

\section{1. - L'interaction ethnique des Tchouvaches avec les Finno-Ougriens : aires, formes et résultats}

\section{1. - Les Tchouvaches et les Maris}

5 Les zones de contact des Tchouvaches avec leurs voisins finno-ougriens se sont mises en place au fil de l'occupation par ces groupes ethniques de l'espace Oural-Volga aux $\mathrm{XVI}^{\mathrm{e}}$-XIX $\mathrm{X}^{\mathrm{e}}$ siècles. C'est ainsi que se sont formés des villages ou des communautés mixtes et que, dans les régions de plus intense interaction, on a pu constater l'existence de mariages mixtes. Au cours de ces processus, les différences culturelles se sont estompées et on a vu émerger des situations de bilinguisme et de participation commune à la vie sociale, aux fêtes et aux rituels.

Historiquement, les contacts avec les Maris ont eu lieu dans le nord de la Tchouvachie actuelle, mais ils ont été limités, surtout, aux régions du nord-ouest de la République et au raïon mari des collines du Mari El qui les jouxte: ce sont les zones habitées par les hauts Tchouvaches et par les Maris des collines (Kozlova, 1958, p. 153-157). Avec les Maris des plaines, les Tchouvaches sont en contact surtout dans le raïon de Zvenigorsk du Mari El (dans les villages de Čuvaš-tary, de Trojary, de Semenovka, etc.; Ivanov, 1999, p. 75).

7 Les Tchouvaches et les Maris étaient voisins dans les communautés, ils se mariaient entre eux, ils étaient en relations amicales (тус кил), ce qui se manifestait par des visites des uns aux autres, par des cadeaux, par l'aide réciproque dans les affaires économiques, par la tenue de fêtes communes. Dans la zone de contact, le bilinguisme tchouvacho-mari était fréquent. Ces relations aussi bien proches qu'intenses ont abouti à la formation d'une couche de traditions ethnoculturelles communes, ce qui apparait très clairement dans les rituels calendaires et familiaux ou dans le costume féminin. $\mathrm{Ce}$ dernier est identique chez les femmes tchouvaches viljars et chez les Maries des 
collines. Dans les mariages mixtes, les femmes changeaient souvent, avec le temps, d'identité: les femmes tchouvaches finissaient par se considérer comme maries (Molotova, 2013, p. 141-143).

8 En dehors de leur territoire principal, les Tchouvaches entrent en contact avec les Maris dans le nord-ouest du Bachkortostan, à savoir dans les raïon de Bakaly (conseil rural de Dijaševo), de Čekmaguš (conseil rural de Jumaševo) et de Šaran (conseils ruraux de Mičurinsk, de Pisarevo et de Sakty).

9 On peut analyser un exemple des relations tchouvacho-maries sur le modèle du village de Jumaševo de la République du Bachkortostan du raïon Čekmaguš. En réalité, le village se compose d'un village tchouvache, Jumaševo, fondé par des Tchouvaches en 1812 , et d'un village mari (teptjar) qui avait émergé, d'après les documents d'archives, dès 1635 (GARB, p. 1). À l'heure actuelle, la plus grande partie de la population est constituée de Tchouvaches $(78,2 \%)$; les Maris représentent $17,2 \%$. L'histoire des relations entre Maris et Tchouvaches date de plus de 200 ans, au cours desquels s'est formée une position dominante de la langue et de la culture tchouvaches, alors que les particularités territoriales et religieuses de la minorité marie se maintenaient. Les Maris vivent dans «leur " partie de Jumaševo - Mari kassi ${ }^{1}$, qui a gardé pour la conscience de ses habitants l'appellation historique Pajkil't ${ }^{2}$ (en russe Bajgil'dino ${ }^{3}$-, ils ont leur cimetière et pratiquent la religion ethnique.

Une des formes d'interaction entre les groupes est le mariage interethnique, considéré de part et d'autre comme la norme. Plus de la moitié des mariages mixtes dans le village $(52,7 \%)$ ont lieu entre Tchouvaches et Maris. Les autres sont des mariages avec des Bachkirs, des Russes et des Tatars. Mais la part des mariages mixtes est faible si on la compare à celle des mariages monoethniques $(28 \%)$. La nationalité des enfants des familles mixtes est déterminée la plupart du temps par celle du père, même si dans certaines jeunes familles, d'après les registres, elle a été établie sur la base de celle de leur mère tchouvache ${ }^{4}$.

11 La «tchouvachisation » des Maris tient tout d'abord à la prédominance numérique des Tchouvaches. Elle commence dès l'enfance : à l'école, les enfants maris, mais aussi les enfants tatars des villages voisins, assimilent le tchouvache. Les élèves de l'école locale, y compris les Maris et les Tatares des villages voisins, passent obligatoirement l'examen de tchouvache. Les particularités des interconnexions des Tchouvaches et des Maris avec la population d'autres ethnies ont conduit à la formation au village de Jumaševo d'une stratification suigeneris (en ordre croissant de prestige) qui se présente de la sorte : le mari - le tchouvache - le tatare / le bachkir / le russe (TTA, 2006 a, Ivanova, Sokolov).

\section{2. - Les Tchouvaches et les Oudmourtes}

12 Les contacts entre Tchouvaches et Oudmourtes sont particulièrement intenses au sud-est du Tatarstan et dans les raïon du nord-ouest du Bachkortostan. Dans le raïon de Bavli en République du Tatarstan, ils ont vécu ensemble dans deux villages (Potapovo-Tumbarla et Udmurtskie Tašly) et ont été voisins dans les conseils ruraux dont ces deux villages composent les centres (ATD, p. 46).

13 Le village Udmurtskie Tašly est un exemple illustrant bien les relations tchouvacho-oudmourtes. Les contacts, anciens et intenses, ont permis une 
" oudmourtisation » partielle des Tchouvaches de ce village qui y représentent une minorité ethnique ( $8,7 \%$ de la population) :

Nous ne nous considérons plus comme tchouvaches. Avant, les Tchouvaches étaient tchouvaches. Ils gardaient leur religion. Ils parlaient tchouvache... Ils n'étaient pas nombreux... Maintenant - il y a dix maisons. Il y a des Tchouvaches qui ne parlent pas tchouvache. Nous vivons avec des Oudmourtes. Soit le mari soit la femme sont oudmourtes. C'est pourquoi nous parlons oudmourte. Nous nous sommes mélangés... Qu'on soit ar ou tchouvache, on vit comme des ars.

(TTA, 2006 b, Ivanova, Maksimova)

14 Les Tchouvaches maîtrisent l'oudmourte mieux que le tchouvache, parce que, aussi bien dans les familles mixtes que, souvent, dans les familles tchouvaches, l'oudmourte était la principale langue de communication :

Nous parlons oudmourte depuis notre enfance. C'est même plus facile de parler oudmourte. Nous traduisons les mots oudmourtes en tchouvache. Nous pensons en oudmourte. Même en discutant avec toi (avec moi - E.Ja ${ }^{5}$ ). En tchouvache, nous ne parlons pas correctement. Les Tchouvaches parlent une belle langue!

(TTA, 2006 b, Ivanova, Maksimova)

15 La famille de V.A. Ivanova nous permet de suivre le mécanisme de l'« oudmourtisation » des Tchouvaches du village. Valentina Alekseevna connaît les deux langues depuis qu'elle est enfant, puisque dans sa famille on ne parlait que tchouvache («Grand-père ne permettait pas qu'on parle oudmourte la maison »), mais dans le village tous, y compris les Tchouvaches, parlaient oudmourte. Elle a épousé un Oudmourte, et à la maison n'a plus parlé qu'oudmourte. Son fils maîtrise l'oudmourte et le russe, et ne connaît que très peu le tchouvache; il a épousé une Oudmourte, et les petits-enfants de Valentina ne connaissent que l'oudmourte et le russe. Dans la famille, on parle au quotidien oudmourte. Il est à noter que cette famille vit dans la zone tchouvache du village. Le passage à l'oudmourte y est ainsi lié aux mariages interethniques, qui ont conduit à une assimilation non seulement linguistique, mais aussi ethnique des Tchouvaches (TTA, 2006 b, Ivanova, Maksimova).

16 L'exemple de la famille de K.I. Afanas'eva (née en 1921) présente un autre modèle de pénétration de l'oudmourte dans une famille tchouvache. Le fils de Klavdija Ivanovna comprend, mais ne parle pas, le tchouvache; il a épousé une Tchouvache du raïon d'Aksubaevo (Tatarstan). Au début, le couple parlait russe, jusqu'à ce que l'épouse assimile l'oudmourte. On trouve maintenant dans cette famille trois langues de communication: Klavdija Ivanovna parle tchouvache avec sa belle-fille, oudmourte avec son fils, celui-ci parle russe avec sa femme, mais parfois aussi oudmourte, et ils parlent oudmourte avec les enfants. Et cette famille, d'après les documents officiels, est considérée comme tchouvache. L'une des petites-filles a épousé un Oudmourte, mais ses enfants parlent russe (TTA, 2006 b, Afanas'eva). Dans ce cas, nous assistons à une assimilation linguistique des Tchouvaches mais accompagnée du maintien de leur conscience ethnique.

17 Le principal canal de l'interaction des groupes ethniques est les contacts matrimoniaux et les innombrables liens de parenté directe des habitants des villages. Il est à noter que les Tchouvaches se marient aussi bien avec des Oudmourtes baptisés qu'avec des Oudmourtes non baptisés, obstacle à la communication, même si en cas de mariage la règle veut que la mariée adopte la religion de son mari. Ainsi, la population tchouvache est intégrée dans les deux groupes confessionnels, au cœur desquels les Oudmourtes dominent numériquement. Au village d'Udmurtskij Tašly, les différences 
confessionnelles comptent davantage que les différences ethniques. C'est sans doute la raison pour laquelle le cimetière rural - un seul pour tout le village - est divisé en deux (partie chrétienne et partie païenne) sur la base du principe religieux et non ethnique. La plupart des Tchouvaches, tradition oblige, sont enterrés dans la partie chrétienne. C'est d'ailleurs ce même critère qui détermine l'organisation territoriale du village - les baptisés et les non-baptisés vivent à des extrémités différentes du village (TTA, 2006 b, Ivanova, Maksimova).

Les choses se présentent autrement dans le village voisin de Potapovo-Tumbarla où la suprématie numérique revient aux Tchouvaches (60,2 \% de Tchouvaches pour 13,4\% d'Oudmourtes). Les mariages interethniques, la domination de la composante tchouvache dans le profil socioculturel du village (l'école a été reconnue comme une école nationale tchouvache; dans les activités de la population, c'est le folklore tchouvache qui est prédominant, le fonds de la bibliothèque est enrichi de littérature tchouvache) ont conduit à une assimilation progressive, linguistique et ethnique, des Oudmourtes. Les spécificités de la répartition territoriale de la population (les Tchouvaches et les Oudmourtes occupent des parties différentes du village) n'empêchent pas d'avoir des relations amicales - de voisinage, d'amitié et de famille. Souvent les relations se nouent dans l'enfance et permettent une perception positive de la spécificité ethnoculturelle de chaque groupe. Le caractère pacifique et amical des relations apparaît bien dans le fait que tout le monde est enterré dans le même cimetière (TTA, 2006 b).

Si les différences au quotidien n'ont pas conduit à de sérieuses contradictions sur le terrain ethnique, les dissensions religieuses entre les Tchouvaches orthodoxes et les Oudmourtes - officiellement baptisés, mais qui ont gardé des éléments de leur religion dans leur culture - ont émergé dans la vie sociale, notamment à l'occasion du rétablissement d'une église dans le village au début des années 2000. Alors que les Tchouvaches étaient les initiateurs du projet et ses partisans les plus passionnés, les Oudmourtes chrétiens n'y ont guère été associés (TTA, 2006 b, Odnodvorceva).

Les contacts des Tchouvaches avec les Oudmourtes et les Maris dans l'Oural occidental ont été limités aux rares cas de coexistence dans des villages voisins (par exemple, dans le conseil rural de Sukkulovo, raïon d'Ermekeevo, Bachkortostan), de quelques mariages mixtes, de relations d'amitié ou entre collègues, et n'ont pas eu d'effet particulier sur le profil ethnoculturel des groupes en question (TTA, $2006 \mathrm{c}$ ).

\section{3. - Les Tchouvaches et les Mordves}

Dans la zone Oural-Volga, les interactions entre Tchouvaches et Mordves ont une tout autre ampleur. Au cœur du territoire tchouvache, elles étaient limitées à quelques villages dans la partie orientale des Tchouvaches anatris (sud-est de la République de Tchouvachie, raïon du nord de l'oblast' d'Uljanov et raïon de la rive droite du Tatarstan). Sur le territoire de la République de Tchouvachie, les Tchouvaches sont voisins des Mordves dans les raïon de Batyrevo (conseil rural de Bahtigildino), d'Ibresi (conseil rural de Malye Karmaly), de Poreckoe (conseil rural de Nikulino) et de Semurša (conseil rural de Trehbaltaevo ; Ivanov, 1997, p. 162-164).

Les relations avec les Mordves ont été historiquement particulièrement significatives pour les groupes territoriaux des Tchouvaches proches de la Volga (raïon de la rive droite des oblast' d'Uljanovsk et de Saratov), de la Kama (raïon du sud du Tatarstan et du 
nord de l'oblast' de Samara), d'outre-Volga (nord-est et est de l'oblast' de Samara) et de l'Oural (oblast' d'Orenburg et de Bachkortostan). Dans ces régions, Tchouvaches et Mordves ont vécu ensemble dans 22 villages.

Les interactions entre Tchouvaches et Mordves se manifestaient historiquement sur la base de mariages mixtes, de villages mixtes et de communautés foncières, de contacts quotidiens, auxquels, à l'époque soviétique, est venue s'ajouter la proximité sur les lieux de travail ainsi que dans les espaces socioculturels communs tels que le jardin d'enfants, l'école, l'hôpital, le conseil rural, etc. : de manière générale, elles ont eu des effets considérables sur l'évolution ethnoculturelle des deux groupes. Le comportement des membres des deux groupes ethniques était guidé par les circonstances, par les stéréotypes ainsi que par toute une série de facteurs (linguistiques, religieux, sociaux) caractérisant la distance entre les deux groupes. En raison de leur christianisation précoce et de leur russification massive, les Mordves, dans la perception des Tchouvaches, étaient souvent rapprochés des Russes (Ivanov, 2005, p. 276). Examinons les traits caractéristiques de l'interaction entre Tchouvaches et Mordves en passant en revue les zones de contact.

24 Au début, les contacts des Tchouvaches avec les Mordves dans les zones à proximité de la Volga étaient des relations de voisinage. Depuis le milieu du XvIII ${ }^{e}$ siècle, il se trouvait dans cette région huit villages communs avec des Mordves, où avaient lieu des mariages interethniques, qui représentaient en moyenne 8-9\% de la totalité (RGADA). Mais, à partir de la seconde moitié du XIX $x^{e}$ siècle, ils se sont également développés sur une base religieuse et ont été à l'origine de la diffusion des pratiques vieilles-croyantes parmi les Tchouvaches. C'est ainsi que la communauté de vieux-croyants du groupe de Tuarma de l'uezd de Karsin dans le gouvernement de Simbirsk comprenait les villages tchouvaches de Kargino, Verhnjaja Tuarma, Čuvašskaja Rešetka, Homuter', dont les habitants étaient inscrits dans un cercle de mariages commun avec le village voisin mordve de Konečnjaevka. On trouvait aussi des vieux-croyants par petits groupes dans les villages proches de Muhino : Nižnjaja Tuarma et Sadovyj. Les « Kalagurs », comme la population appelait les vieux-croyants, ne se mariaient qu'à l'intérieur de leur communauté religieuse, étendant et émoussant par là même les frontières ethniques. Ainsi s'est formée ici une communauté confessionnelle superethnique des vieuxcroyants spasovcy (TTA, $2006 \mathrm{~d}$ ). Des processus analogues ont pu être notés dans la paroisse de Kalamantaevo de l'uezd de Hvalinsk du gouvernement de Saratov : dans les villages d'Eremkino et de Staraja Lebežajka près de 550 Tchouvaches vieux-croyants vivaient avec les Mordves (ISH, p. 781; Nikolsky, 2008, p. 472-473). L'adoption du rite vieux-croyant a conduit à la transformation du profil ethnoculturel des peuples, elle a changé la sphère rituelle et quotidienne de leur existence, replaçant des éléments traditionnels par de nouveaux, empruntés à la culture russe et aux pratiques des vieux-croyants (Iagafova et al., 2010, p. 194). Ainsi le signe de croix à deux doigts, les icônes en cuivre, la lestovka, la littérature des vieux-croyants et leur anthroponymie se sont répandus et enracinés chez les Tchouvaches vieux-croyants (Iagafova et al., 2010, p. 190).

Les contacts dans la zone d'outre-Volga, dans les uezd de Buguruslan et de Buzuluk du gouvernement de Samara, sont attestés depuis l'occupation de cette aire dans la seconde moitié $d u \mathrm{XVIII}^{\mathrm{e}}$ siècle et le premier tiers du $\mathrm{xIX}^{\mathrm{e}}$ siècle. Les Tchouvaches cohabitaient avec les Mordves dans 6 villages et étaient leurs voisins dans 27 volost (RGIA, STSG). Au XIXe siècle, les Mordves étaient, avec les Russes, les voisins les plus 
proches des Tchouvaches (Kappeler, 1982, p. 492). Au Xxe siècle, les Tchouvaches étaient voisins des Mordves dans sept villages de cette région (Ivanov, 1999, p. 94-101, 160-161).

Le voisinage et l'intensité des contacts ont permis la formation d'éléments semblables dans la culture des deux peuples. Les femmes tchouvaches de Buzuluk, comme les Mordves, portaient de larges colliers de perles multicolores et des parures bariolées (REM). On trouve des éléments semblables dans le rite nuptial; par exemple, la pratique de "regarder dans le petit poêle ${ }^{6}$ ", celle de décorer "la beauté de la jeune fille $^{7} »$, celle d'aller chercher de l'eau, de transporter la mariée dans un chariot couvert, et aussi celle du «prix du lait de la mère de la mariée » et celle des jeux érotiques du deuxième jour de la noce. Dans les rites funéraires et commémoratifs, on trouve des pratiques analogues comme l'ouverture d'une « fenêtre » sur le couvercle ou la paroi du cercueil, l'utilisation d'un brancard et d'objets métalliques pour la purification après contact avec le défunt, la pratique de faire des présents aux participants au rituel, les dates de commémoration, etc. On note aussi une analogie structurelle dans les rites calendaires (TTA 2000 ; Zorin, 2001, p. 69, 117 ; MZ, 1984, p. 106-117 ; Iagafova, 2007, p. 301-306).

Dans la région de l'Oural, dans les uezd occidentaux et centraux du gouvernement d'Ufa et dans ceux du nord du gouvernement d'Orenbourg, les Mordves vivaient au XVIII ${ }^{e}$ siècle à proximité des Tchouvaches dans deux villages en tout et pour tout. Au milieu du XIX ${ }^{e}$ siècle ce nombre était monté à cinq (Naumkino, Zirgan, Bižbuljak, Kurmazi, Bazlyk) et, à la fin du xx siècle, à huit (NPB ; Ivanov, 1999, p. 140-151). Malgré une communication plutôt intense dans les relations entre Tchouvaches et Mordves dans cette région, on ne note pas d'influence réciproque sensible. Au contraire, même dans les villages mixtes, les groupes ethniques ont continué au fil des siècles à vivre dans un isolement absolu.

On peut prendre pour exemple d'un voisinage datant de plus de 200 ans le village de Naumkino du raïon d'Aurgazy, au Bachkortostan. Malgré une ancienne cohabitation (depuis le xVIII ${ }^{e}$ siècle) dans le même village et la communauté confessionnelle (les deux groupes sont orthodoxes) il n'y a pas eu de mariages mixtes jusqu'aux années 1960. On n'a pas observé non plus de sérieux conflits dans les rapports de voisinage mais on constate une hostilité mutuelle latente jusqu'à aujourd'hui dans les stéréotypes et dans la mise en place des mariages. Ainsi, les Mordves considèrent les Tchouvaches comme "malins, dissimulés » et ils se voient, corrélativement, comme "un peuple ouvert ", plus pragmatique, ce qui se manifeste par exemple dans la tendance à quitter le village pour aller s'installer en ville («Les Mordves sont plus intelligents, plus civilisés, c'est pourquoi ils partent en ville »). Les Tchouvaches, pour leur part, expliquent ce fait par la paresse des Mordves, leur refus de travailler la terre ( Les Mordves aiment mieux la ville [...], faire des journées de travail cela ne rapporte pas, alors que les Tchouvaches ont toujours été travailleurs et modestes [...], il y a peu de Mordves qui ont des décorations, les tractoristes primés sont surtout tchouvaches»). Parmi les traits positifs de leurs voisins, les Tchouvaches estiment que les Mordves sont autonomes et capables de se défendre («Les Mordves ont la langue bien pendue »), ils reconnaissent leur musicalité. Les Mordves préfèrent choisir un partenaire parmi les gens de leur nationalité et, si un Mordve épouse une Tchouvache, on va dire qu'il n'a même pas pu trouver une Mordve. Pourtant, malgré tout, les mariages entre Mordves et Tchouvaches sont préférés dans les deux groupes aux mariages avec des Tatares (TTA, 2007 a, Afanas'eva, Afanas'ev). 

bilinguisme tchouvacho-mordve. Mais le niveau de connaissances diffère : si une partie considérable des Mordves parlent tchouvache de manière grammaticalement correcte et presque sans accent, les Tchouvaches reconnaissent et comprennent le mordve. C'est probablement une manifestation de la prédominance numérique des Tchouvaches dans le village. Dans la communication entre eux, les deux groupes utilisent le plus souvent le russe, que traditionnellement les Mordves maîtrisent mieux que les Tchouvaches, lesquels historiquement parlaient parfaitement le tatar (TTA, 2007 a). permis de renforcer la position de la langue et de la culture mordves : depuis, la maison de la culture met l'accent sur les pratiques folkloriques mordves. Mais il y a toujours des Tchouvaches qui participent à toutes les manifestations au sein des groupes folkloriques.

31 En situation de minorité ethnique, par exemple au village de Timjaševo dans le raïon de Šentala, oblast' de Samara, les Tchouvaches ont été assimilés par les Mordves. C'est bien le facteur linguistique qui l'a permis - la priorité donnée au mordve (à l'erza) en conséquence de la prédominance numérique (à l'heure actuelle, les Mordves sont plus de 70 \%). D'après les habitants, « les Tchouvaches de Timjaševo parlent bien mordve, ils parlent moins bien le tchouvache; ils parlent davantage en mordve ». Même dans les familles tchouvaches, les jeunes époux parlent tchouvache à leurs parents, mais entre eux ils se parlent soit en mordve soit en russe. En même temps, les Mordves ne parlent pas tchouvache. Mais même dans cette situation, les deux groupes conservent leur animosité réciproque, qui se reflète dans les auto- et hétérostéréotypes. Ainsi les Mordves considèrent les Tchouvaches comme mesquins et se voient comme simples, mais préférant les beuveries et les bagarres au travail. Ce dernier trait, les Mordves doivent le reconnaître, s'est diffusé aussi chez les Tchouvaches de Timjaševo, à la différence desquels les Tchouvaches du village voisin de Salejkino sont considérés comme plus travailleurs. Les Tchouvaches aussi se considèrent comme travailleurs et simples, mais de plus, à la différence des Mordves, ils ont de l'initiative :

МӑКШӑсЕНЕ «ЗАРЯДИТ» ТУМАЛЛА, А ЧӑВАШ ШУТЛАТ КАС̧ХИНЕ, ИРХИНЕ МӗН ТУМАлЛИНЕ (Les Mordves doivent être «rechargés", mais les Tchouvaches pensent dès le soir à ce qu'ils vont faire le lendemain matin ; TTA, 2008).

Dans ce cas comme dans le cas précédent, le choix de stéréotypes reflète aussi bien le comportement réel des membres des groupes ethniques que l'attitude subjective de la part des voisins. En même temps ils confirment l'existence de frontières ethniques entre les groupes et leur portée dans la communication interethnique aujourd'hui.

\section{2. - La culture des rituels et des fêtes comme un espace d'interaction entre les Tchouvaches et les Finno-Ougriens}

Un des indicateurs significatifs du niveau d'interaction des Tchouvaches avec leurs voisins finno-ougriens est le fonctionnement du système des fêtes et des rituels dans les groupes ethniques - avec la participation et le caractère de celle-ci aux rituels, le passage d'éléments d'un groupe à l'autre, l'existence de traditions cérémonielles communes, etc. 

la tenue commune aux deux groupes des ensembles de fêtes du nouvelan, de mardi gras, de Pâques, de la Trinité, ainsi que les journées commémoratives des parents morts. Cette tendance apparaît fort clairement dans la culture des Tchouvaches à proximité de la Volga et elle se développe dans le contexte d'une grande proximité avec les Mordves et avec les Russes ${ }^{8}$. Le point culminant des fêtes du printemps/été ВЕСНА ӑ АТНИИ (littéralement : « accompagnement du printemps ») et la mise en place des «maisons de Noël » sont analogues dans l'appellation, dans le mode d'organisation et dans le contenu avec les rituels correspondants chez les Mordves (NA CHGIGN ; TTA, 1993, 1994 ; Matveyev, 2001, p. 161). Jusqu'au milieu du xxe siècle, dans certains villages, on organisait des cérémonies et des fêtes communes des jeunes Mordves et des jeunes Tchouvaches (SSCH, 2004, p. 45).

Dans d'autres localités de contact entre Tchouvaches et Finno-Ougriens étudiées précédemment, les caractéristiques des corrélations entre les groupes sont aussi visiblement projetées sur la culture rituelle et festive. Je prendrai un exemple d'une cohabitation longue et intense entre Tchouvaches et Mordves à Timjaševo : cela, du point de vue des habitants, leur a permis de niveler les rites nuptiaux, funéraires et de commémoration. Certains rituels avaient lieu avec la participation des deux groupes. Ainsi, les Tchouvaches participaient à l'« accompagnement du printemps » à la Trinité, et les Mordves, aux cérémonies tchouvaches učuk et ujav; ils pratiquaient ensemble la divination de Noël et faisaient de la luge à mardi gras (TTA, 2008).

participation mutuelle aux rituels fait partie aussi des pratiques communes à Udmurtskie Tašly; plus précisément, les Oudmourtes invitaient les Tchouvaches au banquet suivant les sacrifices (sans jamais leur permettre d'assister à la cérémonie sacrificielle elle-même) lors des fêtes d'automne. On peut relever des cas significatifs de visites réciproques lors du cycle pascal et du cycle des commémorations de la Trinité. Les Oudmourtes non baptisés commencent à fêter bydžynal ${ }^{9}$ dès le vendredi de la semaine sainte et invitent les Tchouvaches, qui les accueilleront le dimanche à leur tour. Les Tchouvaches se rendent deux fois au cimetière à la Trinité : le samedi, comme tous les Tchouvaches, et aussi le mercredi avec les Oudmourtes. Ils s'expliquent de la sorte :

Nous y allons aussi le mercredi parce que les parents sont là les bols vides. Nous faisons comme les Ars et comme les Tchouvaches. Nous sommes un peuple mélangé.

(TTA, 2006 b, Maksimova, Ivanova)

L'une des conséquences de ce "mélange », c'est l'entraide active des Oudmourtes et des Tchouvaches dans les rituels de commémoration, ce que relèvent surtout les habitants des villages :

Ici on va tous s'aider mutuellement aux enterrements et aux commémorations. On ne fait pas attention, tous, Oudmourtes, Tchouvaches, Tatares, on apporte de la nourriture et de la boisson.

(TTA, 2006 b, Afanas'eva)

Le rapprochement des Tchouvaches et des Oudmourtes a commencé au milieu des années 1920. Avant, les deux groupes existaient de manière relativement isolée, et les Tchouvaches, malgré le fait qu'ils étaient peu nombreux, préféraient effectuer leurs rituels dans leur propre cercle :

Les Tchouvaches vivaient de leur côté, ils ne se mélangeaient pas aux Ars, ils faisaient leurs fêtes séparément. 
39 À partir des années 1930, la fermeture des groupes ethniques a commencé à s'effriter, mais elle s'est maintenue jusqu'à la fin des années 1980 ; maintenant, d'après l'expression de K.I. Afanas'eva, les Tchouvaches « se sont dispersés, ils se sont mélangés avec les Ars » (TTA, 2006 b, Afanas'eva).

40 L'importance du facteur confessionnel ne se remarque pas seulement dans le cas de la tradition orthodoxe. Dans ce même village de Timjaševo, les rituels animistes des Tchouvaches ont stimulé les Mordves à organiser leurs ozk $k^{10}$ avant la fin du XIX siècle. La préservation de toute une série de rituels animistes (restent en fonctionnement tous les principaux rituels de l'ensemble animiste : le nartukan, le çavarni, le munkun, le çiměk, l'avtan sări) des Tchouvaches du village Staroe Jumaševo a été stimulée par le maintien des traditions animistes de la part d'une partie de la population marie. À Udmurtskie Tašly, sur l'exemple des Oudmourtes animistes ${ }^{11}$, les Tchouvaches orthodoxes commémorent leurs ancêtres le lundi de Pâques, bien que dans les villages tchouvaches voisins ce rituel ait lieu le mardi de la semaine de Pâques (TTA, 2007 b).

41 Nous avons un bon exemple de syncrétisme religieux et en même temps d'assimilation linguistique des Tchouvaches avec le rituel d'accompagnement des âmes des morts à la fin de la commémoration annuelle des ancêtres à Pokrov (le 14 octobre) et le troisième jour de la «Pâque » à Udmurtskie Tašly. La maîtresse de maison rassemble des petits morceaux de nourriture de la table de la commémoration dans un bol commun (analogie avec le rituel hvyny chez les animistes), l'apporte dans un " endroit propre ", et s'adresse aux esprits des ancêtres en oudmourte, prenant congé et les envoyant « en visite ». Elle laisse le bol dehors pour la nuit et ne le rentre que le lendemain. Un autre bon exemple d'assimilation linguistique est la tradition du chant, notamment des chants rituels (nuptiaux, du nouvel an) chantés sur des mélodies tchouvaches avec un texte qui est un calque de l'original tchouvache. En même temps, les Tchouvaches ont activement assimilé le répertoire des chants oudmourtes (TTA, 2007 b).

L'interaction a souvent lieu également dans le cadre de cérémonies de groupes ethniques et religieux divers: par exemple la fête akatuj/sabantuj rassemble les Tchouvaches et les Tatars, les Bachkirs et parfois aussi les Mordves, par exemple au village de Salejkino : le sabantuj du village accueille les habitants de trois villages, les Tchouvaches orthodoxes de Salejkino, les Tchouvaches chrétiens ou non chrétiens du village voisin de Staryj Afon'kino et les Mordves du village de Podlesnaja Andreevka, qui travaillent tous dans la même coopérative agricole (TTA, 2001). Dans les villages polyethniques, les enterrements et les commémorations, les noces et les autres festivités familiales rassemblent les habitants du village hors appartenance ethnique.

De cette manière, la culture des rituels et des fêtes a joué et joue encore un rôle surtout de consolidation dans la vie des habitants des villages mixtes. En même temps, c'est bien à ces occasions que les fêtes et rituels à coloration ethnique servent de définition ethnoculturelle des groupes en contact et délimitent l'espace culturel entre " le nôtre " et l'« autre ", permettant la formation et le maintien de l'identité ethnique de leurs membres. Par exemple, au village de Naumkino, les Tchouvaches et les Mordves fêtent ensemble les fêtes du nouvel an avec des divinations, mardi gras, Pâques, les rituels de la Trinité; ils partagent aussi certains éléments des rites funéraires et de commémoration (la distribution des cadeaux, des fils, les jours obligatoires de commémoration). Mais en même temps on note aussi des différences dans la culture ethnique de la population. Ainsi, les Tchouvaches reconnaissent comme «leurs " les divinations du nouvel an nartukan, le rituel d'exorcisation des esprits malveillants et de 
purification sĕren, le repas à la famille lors du cycle pascal et la commémoration des ancêtres à l'automne, les rituels de "nourriture " hyvni et la communication avec les ancêtres hutăštarsa accompagnée d'un sacrifice. En même temps ils connaissent bien les traits caractéristiques des rituels funéraires et de commémoration des Mordves ( On ne va aux commémorations des Mordves que s'ils nous invitent ») et voient les Mordves comme plus proches de l'orthodoxie. Les lieux des rituels sont, eux aussi, différents, par exemple celui du rituel pour appeler la pluie: en cas de sécheresse, les Tchouvaches arrosent d'eau une pierre, la čul laša (« cheval de pierre »), qui se trouve à proximité du village sur une colline, alors que les Mordves accomplissent la cérémonie baban' kaša auprès d'une source à l'autre bout du village. Ainsi, les deux groupes ethniques maintiennent des marqueurs significatifs qui les distinguent (TTA, 2007 a).

Les frontières ethnoculturelles entre Tchouvaches et Mordves sont encore davantage préservées au village d'Il'terjakovo, raïn de Karmaskaly au Bachkortostan. Les différences religieuses se manifestent dans l'intégration active et importante des Mordves dans l'orthodoxie sur un fond de maintien, chez les Mordves d'Il'terjakovo, de leur "animisme » : officiellement chez les non-baptisés, et non officiellement mais effectivement chez les orthodoxes. Dans l'ensemble des rituels funéraires et des commémorations des Tchouvaches, il existe jusqu'à aujourd'hui le rituel d'invitation des morts à manger, appelé hyvni, et le repas offert à la famille, où l'on trouve certains éléments des pratiques animistes (par exemple, on met sur la tête du mort d'abord un poteau et seulement après une croix). Un autre élément assez stable est aussi le rituel munkun (la Pâque tchouvache), avec le nesĕl, un repas offert à la famille de la lignée du mari, le gruau obligatoire parmi les plats, etc. (TTA, $2007 \mathrm{~b}$ ).

Parmi les facteurs d'intégration ethnique des différents groupes, il faut mentionner la similitude des modes de vie, des activités économiques et des traditions religieuses, la compétence culturelle et linguistique partagée, l'acceptation des différences ethniques et culturelles. De plus, diverses institutions sociales (groupements, écoles, kolkhozes, etc.) servent de plates-formes d'interaction socioculturelle.

Et pourtant les Tchouvaches ont préservé une distance culturelle par rapport à leurs voisins finno-ougriens : ils sont allés s'installer à des «bouts » différents des villages, ont des cimetières à eux ou une partie réservée dans les cimetières communs, ils ont gardé leurs habitudes, organisé leurs fêtes et rituels tchouvaches, et ils ont donné la préférence aux mariages à l'intérieur de leur communauté ethnique, etc. Les raisons de l'isolement des Tchouvaches par rapport à leurs voisins doivent être cherchées dans les chiffres de la population, qui était suffisante pour sa reproduction en tant que communauté ethnoculturelle: celle-ci était motivée pour maintenir son identité ethnique et culturelle, soutenant une vision positive de la langue et de la culture tchouvaches, et, à l'inverse, développant des stéréotypes négatifs par rapport aux voisins.

\section{Conclusion}

Dans les trois domaines les plus importants (domaines linguistique, ethnoculturel et social), on note dans les relations des Tchouvaches avec les Finno-Ougriens les constantes suivantes :

1) dans la sphère linguistique - existence de bilinguisme (connaissance de la langue du voisin) dans une certaine mesure, avec une tendance au multilinguisme ; 
2) dans la sphère de la culture ethnique - développement de deux tendances contradictoires :

- d'une part, on observe dans la culture religieuse et rituelle, des manifestations de syncrétisme caractérisées par A) la pénétration mutuelle dans la tradition d'éléments de la culture du voisin qui, avec le temps, finissent par être perçus comme «siens »; b) un haut niveau de compétence interculturelle; c) le dépassement de l'isolement religieux des groupes et la tradition de la tenue par les groupes ethniques et ethno-confessionnels de rituels communs,

- mais, d'autre part, on note la tendance à l'individuation des groupes ethniques du point de vue religieux et linguistico-culturel, qui se manifeste dans la structure spatiale des villages mixtes (rues, parties, extrémités différentes), dans l'organisation de l'espace culturel (lieux de culte, cimetières), dans les préférences ethniques dans le choix des amis, des époux et des partenaires ainsi que dans les stéréotypes de l'autre (le voisin);

3) dans la sphère sociale, on note une adaptation réciproque des groupes ethniques et confessionnels à différents niveaux de l'interaction sociale (voisinage, équipes professionnelles, famille, etc.), un haut niveau de mariages mixtes, l'existence de nombreuses familles mixtes, et différentes configurations de rapports de parenté, directs et indirects, variant beaucoup selon les villages.

\section{BIBLIOGRAPHIE}

\section{Sources}

ATD $=$ Subdivisions administratives et territoriales de la République du Tatarstan : recueil statistique, 2005, Kazan (АДМИНИСТРАТИВНО-ТЕРРИТОРИАЛЬНОЕ ДЕЛЕНИЕ РЕСПУБЛИКИ ТАТАРСТАН: СТАТИСТИЧЕСКИЙ СБОРНИК 2005 (4 РЕД.), КАЗАНЬ: ИЗДАТЕЛЬСКИЙ ЦЕНТР ТАТАРСТАНСТАТА).

GARB = Archives d'État de la République du Bachkortostan (ГАРБ ГОСУДАРСТВЕННЫЙ АРХИВ РЕСПУБЛИКИ БАШКОРТОСТАН, ФОНД 1, ОПИСЬ 1, ДЕЛО 2436).

ISH = « Les écoles allogènes du diocèse de Saratov / Registre du diocèse de Saratov (ИНОРОДЧЕСКИЕ ШКОЛЫ В САРАТОВСКОЙ ЕПАРХИИ // САРАТОВСКИЕ ЕПАРХИАЛЬНЫЕ ВЕДОМОСТИ) 1900, № 12.

TTA = Travaux de terrain d'E. Iagafova 1993-2008:

TTA 1993, oblast' de Samara, raïon de Stavropol.

TTA 1994, oblast' de Penza, raïon de Neverkino ; oblast' de Samara, raïon de Šigony.

TTA 2000, oblast' d'Orenburg, raïon de Soročinsk et de Gračëvka.

TTA 2001, oblast' de Samara, raïon de Šentala, Salejkino.

TTA 2006 A, République du Bachkortostan, raïon de Čekmaguševo, Jumaševo (Informateurs : Ivanova V.A., née en 1940, Sokolov V.V., né en 1960). 
TTA 2006 b, République du Tatarstan, raïon de Bavlino, Udmurtskie Tašly (Informateurs : Afanas'eva K.I., née en 1921, Afanas'eva V.K., née en 1959, Ivanova V.A., née en 1931, Maksimova E.N., née en 1945), Potapovo Tumbarla (Informatrice : Odnodvorceva).

TTA 2006 c, République du Bachkortostan, raïon d'ЕРМЕКЕEВo, Sukkolovo.

TTA 2006 d, oblast' d'Uljanovsk, raïon de Veškama, Kočenaevka (Informatrice : Kaliganova F.P., née en 1928) ; Kargino (Informatrice : Židkova K.I., née en 1932).

TTA 2007 a, République du Bachkortostan, raïon d'Aurgazy, Naumkino (Informateurs : Afanas'eva L.E., née en 1941 ; Afanas'ev V.N., né en 1941).

TTA 2007 b, République du Bachkortostan, raïon de Karmaskaly, Il’terjakovo.

TTA 2008, oblast' de Samara, raïon de Šentala, Timjaševo (Informatrice : Trofimova N.V., née en 1929, Mordve)

NPB = Hismatullin A.A., 2002, Les points habités du Bachkortostan (хисмАтуллин, А.А. [ed.] 2002: НАСЕЛЕННЫЕ ПУНКТЫ БАШКОРТОСТАНА. ЧАСТЬ. І. УФИМСКАЯ ГУБЕРНИЯ [1877], УФА: КИТАП).

NA CHGIGN = Archive scientifique de l'Institut tchouvache des sciences humaines (НАучныЙ АРХИВ ЧУВАШСКОГО ГОСУДАРСТВЕННОГО ИНСТИТУТА ГУМАНИТАРНЫХ НАУК, ОТДЕЛ I, ЕДИНИЦА ХРАНЕНИЯ 207, ОТДЕЛ III, ЕДИНИЦА ХРАНЕНИЯ 333, 3176).

RGADA = РГАДА, Archive russe des documents anciens (РОССИЙСКИЙ ГОСУДАРСТВЕННЫЙ АРХИВ ДРЕВНИХ АКТОВ, ФОНД, 350, ОПИСЬ 2, ЧАСТЬ 2, ДЕЛА 3144, 3155.

RGIA = РГИА, Archive nationale historique russe (РОсСИЙСКИЙ ГОСУДАРСТВЕННЫЙ ИСТОРИЧЕСКИЙ АРХИВ, ФОНД 379, ОПИСЬ 1, ДЕЛА 622, 624).

REM = РЭМ, Musée ethnographique russe (РОсСИЙСКИЙ ЭТНОГРАФИЧЕСКИЙ МУЗЕЙ, колЛЕКцИЯ 968, ЕДИНИЦЫ ХРАНЕНИЯ 11, 13, 22, 23).

STSG, 1870 = Anučin E., Rihterov I., Tableaux statistiques du gouvernement de Samara (АНУЧИН Е., РИХТЕРОВ И. [ed.], 1870: СТАТИСТИЧЕСКИЕ ТАБЛИЦЫ САМАРСКОЙ ГУБЕРНИИ. ЧАСТЬ 1. САМАРА: САМАРСКИЙ ГУБЕРНСКИЙ СТАТ. КОМИТЕТ).

\section{Références bibliographiques}

BARTH, 2006 = БАРТ ФРЕДРИК (ed.), ЭТНИЧЕСКИЕ ГРУППЫ И СОЦИАЛЬНЫЕ ГРАНИЦЫ. СОЦИАЛЬНАЯ ОРГАНИЗАЦИЯ КУЛЬТУРНЫХ РАЗЛИЧИЙ (Les groupes ethniques et les frontières sociales. L'organisation sociale des différences culturelles), МОсКВА: НОВОЕ ИЗДАТЕЛЬСТВО.

DANILKO et al., 2010 = ДАНИЛКО ЕЛЕНА СЕРГЕЕВНА, КОРОСТЕЛЕВ АЛЕКСАНДР ДМИТРИЕВИЧ, МОЛОТОВА ТАМАРА ЛАВРЕНТЬЕВА, ПОПОВА ЕЛЕНА ВАСИЛЬЕВНА, САДИКОВ РАНУС РАФИКОВИЧ, ЯГАФОВА ЕКАТЕРИНА АНДРЕЕВНА, «ОПЫТ ИССЛЕДОВАНИЯ ВЗАИМНОЙ СОЦИАЛЬНО-КУЛЬТУРНОЙ АДАПТАЦИИ В ЭТНИЧЕСКИ СМЕШАННЫХ СЕЛЕНИЯХ» (Une expérience de recherche sur l'adaptation socioculturelle mutuelle dans les villages à population ethniquement mixte) in ДЕРЕВЯнКо А.П. , КУДЕЛИН А.Б., ТИшКОВ В.А. (eds.), АДАПТАЦИЯ НАРОДОВ И КУЛЬТУР К ИЗМЕНЕНИЯМ ПРИРОДНОЙ СРЕДЫ, СОЦИАЛЬНЫМ И ТЕХНОГЕННЫМ ТРАНСФОРМАЦИЯМ (L'adaptation des peuples et des cultures à l'environnement naturel et aux transformations sociales et technologiques), москВА: РОсспэн, сс. 344-350.

IAGAFOVA, 2007 = ЯГАФОВА ЕКАТЕРИНА АНДРЕЕВНА, ЧУВАШИ УРАЛО-ПОВОЛЖЬЯ: ИСТОРИЯ ФОРМИРОВАНИЯ И ТРАДИЦИОННАЯ КУЛЬТУРА ЭТНОТЕРРИТОРИАЛЬНЫХ ГРУПП (ХVII-НАЧАЛО ХХ В.) (Les Tchouvaches dans les régions de l'Oural et de la Volga : histoire de leur formation et culture 
traditionnelle des groupes ethnoterritoriaux du XVII siècle au début du XX ${ }^{\mathrm{e}}$ siècle), ЧЕБОКСАРЫ: ЧУВАШСКИЙ ГОСУДАРСТВЕННЫЙ ИНСТИТУТ ГУМАНИТАРНЫХ НАУК.

IAGAFOVA, 2007 = ЯГАФОВА ЕКАТЕРИНА АНДРЕЕВНА, «ЧУВАШИ В МЕЖЭТНИЧЕСКОМ ВЗАИМОДЕЙСТВИИ В ЗАКАМЬЕ» (Les Tchouvaches et les interactions interethniques dans l'outre-Kama), ЭТНОГРАФИЧЕСКОЕ ОБОЗРЕНИЕ, 2010/6, сc. 35-53.

IAGAFOVA et al., 2010 = ЯГАФОВА ЕКАТЕРИНА АНДРЕЕВНА (ed.), ДАНИЛКО ЕЛЕНА СЕРГЕЕВНА, КОРНИШИНА ГАЛИНА АЛЬБЕРТОВНА, МОЛОТОВА ТАМАРА ЛАВРЕНТЬЕВА, САДИКОВ РАНУС РАФИКОВИЧ, ЭТНОКОНФЕССИОНАЛЬНЫЕ МЕНЬШИНСТВА НАРОДОВ УРАЛО-ПОВОЛЖЬЯ (Les minorités ethnoconfessionnelles des peuples de l'Oural et de la Volga), САМАРА: ПГСГА.

IVANOV, 1997 = ИВАНОВ В.П., ЭТНИЧЕСКАЯ КАРТА ЧУВАШИИ (Histoire ethnique de la Tchouvachie), ЧЕБОКСАРЫ: ЧГИГН.

IVANOV, 1999 = ИВАНОВ В.П., ЧУВАШСКАЯ ДИАСПОРА: РАССЕЛЕНИЕ И ЧИСЛЕННОСТЬ. ЭТНОГЕОГРАФИЧЕСКИЙ СПРАВОЧНИК (La diaspora tchouvache : implantation et effectifs), ЧЕБОКСАРЫ: ЧГИГН.

IVANOV, 2005 = ИВАНОВ В.П., ЭТНИЧЕСКАЯ ГЕОГРАФИЯ ЧУВАШСКОГО НАРОДА. ИСТОРИЧЕСКАЯ ДИНАМИКА И РЕГИОНАЛЬНЫЕ ОСОБЕННОСТИ РАССЕЛЕНИЯ (Géographie ethnique du peuple tchouvache. Dynamique historique et spécificités régionales de son implantation), ЧЕБОКСАРЫ: ЧУВАШСКОЕ КНИЖНОЕ ИЗДАТЕЛЬСТВО.

KAPPELER Andreas, 1982, Russlands erste Nationalitäten. Das Zarenreich und die Völker der Mittleren Wolga vom 16. bis 19. Jahrhunderts, Köln/Wien/München: Böhlau.

KOZLOVA, 1958 = КОЗЛОВА К.И., «ОБ ЭТНИЧЕСКИХ СВЯЗЯХ ЧУВАШЕЙ И МАРИЙЦЕВ» (Sur les relations ethniques entre Tchouvaches et Maris), ВЕСТНИК МОСКОВСКОГО УНИВЕРСИТЕТА. ИСТОРИКО-ФИЛОЛОГИЧЕСКАЯ СЕРИЯ, МОСКВА, СТР. 153-157.

KOROSTELEV, 2007 = КОРОСТЕЛЕВ АЛЕКСАНДР ДМИТРИЕВИЧ (ed.), МЫ И КАК УДМУРТЫ, И КАК ЧУВАШИ... ВЗАИМНАЯ СОЦИАЛЬНО-КУЛЬТУРНАЯ АДАПТАЦИЯ В ЭТНИЧЕСКИ СМЕШАННЫХ СЕЛЕНИЯХ

УРАЛО-ПОВОЛЖЬЯ (Nous sommes aussi bien comme les Oudmourtes que comme les Tchouvaches... L'adaptation socioculturelle mutuelle dans les villages ethniquement mixtes de la région de l'Oural et de la Volga), МосКВА: ИЭА РАН.

KOROSTELEV, 2009 = КОРОСТЕЛЕВ АЛЕКСАНДР ДМИТРИЕВИЧ (ed.), ОПЫТ ВЗАИМОДЕЙСТВИЯ И ВЗАИМНОЙ АДАПТАЦИИ В ЭТНИЧЕСКИ СМЕШАННЫХ СЕЛЕНИЯХ УРАЛО-ПОВОЛЖЬЯ (Une expérience d'interaction et d'adaptation mutuelle dans les villages mixtes de la région de l'Oural et de la Volga), москвА: ИЭА РАН.

KOROSTELEV, 2010 = КОРОСТЕЛЕВ АЛЕКСАНДР ДМИТРИЕВИЧ, «МЕЖЭТНИЧЕСКИЕ БРАКИ В ЭТНИЧЕСКИ СМЕШАННЫХ СЕЛЕНИЯХ ПРИУРАЛЬЯ И ПОВОЛЖЬЯ (ПО МАТЕРИАЛАМ ЭКСПЕДИЦИЙ 2006, 2007, 2008 Гг.) (Les mariages interethniques dans les villages ethniquement mixtes de la région de l'Oural et de la Volga), ЭТНОГРАФИЧЕСКОЕ ОБОЗРЕНИЕ, 2010/6, сc. 22-34.

MATVEEV, 2001 = МАТВЕЕВ ГЕОРГИЙ БОРИСОВИЧ, «ЭТНОКУЛЬТУРНЫЕ ПРОЦЕССЫ У ЧУВАШЕЙ СИМБИРСКОГО И САРАТОВСКОГО ПРЕДВОЛЖЬЯ (ПО МАТЕРИАЛАМ ЭКСПЕДИЦИИ 2000 Г.)» (Les processus interculturels chez les Tchouvaches des régions de Simbirsk et de Saratov, d'après les matériaux de l'expédition de 2000), in АКБУЛАТОв И.М. И ДР. (ed.), ПРОБЛЕМЫ КУЛЬТУРОГЕНЕЗА НАРОДОВ ВОЛГО-УРАЛЬСКОГО РЕГИОНА, УФА: ЦЕНТР ЭТНОЛОГИЧЕСКИХ ИССЛЕДОВАНИЙ УРО РАН. MOLOTOVA, 2013 = МОЛОТОВА ТАMAРА ЛАВРЕНТЬЕВА, «МЕЖЭТНИЧЕСКИЕ СВЯЗИ ЧУВАШЕЙ И МАРИЙЦЕВ» (Les relations interethniques des Tchouvaches et des Maris), ПРОБЛЕМЫ 
ЭТНОКУЛЬТУРНОГО ВЗАИМОДЕЙСТВИЯ В УРАЛО-ПОВОЛЖЬЕ: ИСТОРИЯ И СОВРЕМЕННОСТЬ. СБОРНИК

СТАТЕЙ, САМАРА: ПГСГА, СТР. 141-143.

$\mathrm{MZ}$ = КОзЛОВ ВИКТОР ИВАНОВИЧ (ed.), 1984, МОРДВА ЗАВОЛЖЬЯ (Les Mordves de la région d'outre-Volga), САРАНСК: МОРДОВСКОЕ КНИЖНОЕ ИЗДАТЕЛЬСТВО.

NIKOLSKY, 2008 = НИКОЛЬСКИЙ НИКОЛАЙ ВАСИЛЬЕВИЧ, СОБРАНИЕ СОЧИНЕНИЙ. ТОМ III (E Euvres complètes, tome III), ТРУДЫ ПО ИСТОРИИ, КУЛЬТУРЕ И СТАТИСТИКЕ НАРОДОВ ВОЛГО-УРАЛЬЯ И СИБИРИ, ЧЕБОКСАРЫ: ЧУВАШСКИЙ ГОСУДАРСТВЕННЫЙ ИНСТИТУТ ГУМАНИТАРНЫХ НАУК.

POPOVA, 2010 = ПОПОВА ЕЛЕНА ВАСИЛЬЕВНА, «УДМУРТЫ В ЭТНИЧЕСКИ СМЕШАННЫХ СЕЛЕНИЯХ УРАЛО-ПОВОЛЖьЯ: ОПЫТ СОСЕДСТВА» (Les Oudmourtes dans les villages ethniquement mixtes de la région de l'Oural et de la Volga : une expérience de voisinage), ЭТНОГРАФИЧЕСКОЕ ОБОЗРЕНИЕ, 2010/6, СТР. 66-80.

SADIKOV, 2010 = САДИКОВ РАНУС РАФИКОВИЧ, «ПРОЦЕССЫ МЕЖКОНФЕССИОНАЛЬНОГО ВЗАИМОДЕЙСТВИЯ В ЭТНИЧЕСКИ СМЕШАННЫХ СЕЛЕНИЯХ УРАЛО-ПОВОЛЖЬЯ: ИСТОРИЯ И СОВРЕМЕННЫЕ ТЕНДЕНЦИИ РАЗВИТИЯ» (Les processus d'interaction interconfessionnelle dans les villages ethniquement mixtes de la région de l'Oural et de la Volga : histoire et tendances actuelles), ЭТНОГРАФИЧЕСКОЕ ОБОЗРЕНИЕ, 2010/6, СТР. 9-22.

SSCH, 2004 = КОНДРАТЬЕВ МИХАИЛ ГРИГОРЬЕВИЧ (ed.), СИМБИРСКО-САРАТОВСКИЕ ЧУВАШИ. ЧЕБОКСАРЫ (Les Tchouvaches de la région de Simbirsk et de Saratov. Tchéboksary), ЧУВАШСКИЙ ГОСУДАРСТВЕННЫЙ ИНСТИТУТ ГУМАНИТАРНЫХ НАУК.

ZORIN, 2001 = ЗОРИН НИКОЛАЙ ВЛАДИМИРОВИЧ, РУССКИЙ СВАДЕБНЫЙ РИТУАЛ (Le rituel nuptial russe), МОСКВА: НАУКА.

\section{NOTES}

1. En mari : МАРИ КАССИ.

2. En mari : ПАЙКИЛЬт.

3. En russe : БАЙГИЛЬДИНо.

4. Observations de l'auteur sur la base des registres du village de Jumaševo : FM, 2006 a.

5. L'auteure de cet article est elle-même tchouvache et elle mène ses entretiens en tchouvache (N.d.R.).

6. Rite d'origine mordve qui consiste pour la mariée à inspecter les biens du marié (N.d.T.).

7. Les tresses (N.d.T.).

8. $\mathrm{Au} \mathrm{Xx}^{\mathrm{e}}$ siècle, les Mordves ont vécu avec les Tchouvaches dans neuf villages de la Volga de Simbirsk et Saratov (Čuvašskaja Kulatka, Staraj Lebežajka, Kalmantaj, Mordovskij Šmalak, Sosnovka, Živajkino, etc.), et avec les Russes dans trois d'entre eux (SSCH, p. 44 ; Ivanov, 1999, p. 107, 113-116).

9. En oudmourte : Быдзынал, litt. « le grand jour », la fête correspondant à la Pâque orthodoxe (N.d.T.).

10. Fête traditionnelle erza.

11. En oudmourte : АРлА (arla). 


\section{RÉSUMÉS}

Cet article examine une expérience de contacts interculturels des Tchouvaches, peuple parlant une langue turcique, avec les peuples finno-ougriens (Maris, Oudmourtes, Mordves) dans différentes régions de l'aire Oural-Volga. Cette étude repose sur les travaux de terrain de l'auteur. L'analyse part de la vision de l'ethnicité comme forme d'organisation sociale de la différence culturelle, qui définit le caractère de l'interaction interethnique, ses formes, ses mécanismes et ses effets. On constate certaines régularités dans les rapports de Tchouvaches avec les Finno-Ougriens. Dans la sphère linguistique, il existe un bilinguisme tendant à se transformer en multilinguisme. Dans la sphère de la culture ethnique, on note un syncrétisme dans la culture des rituels religieux, reconnaissable à la pénétration, dans les ensembles traditionnels, d'éléments provenant de la culture des voisins qui, avec le temps, sont appropriés, un haut niveau de compétences interculturelles, le dépassement de l'éloignement religieux des groupes et la tradition de tenue de rituels communs à des groupes ethniques et ethnoconfessionnels. On constate en même temps une tendance à l'isolement religieux et linguisticoculturel des groupes ethniques qui se manifeste dans la structure territoriale des villages mixtes, dans l'organisation de l'espace culturel, dans les préférences ethniques dans les contacts interethniques et dans les stéréotypes de perception des voisins. La sphère sociale est caractérisée par l'adaptation socioculturelle mutuelle des groupes ethniques et confessionnels à différents niveaux de l'interaction sociale, un haut niveau de mariages interethniques, l'existence d'une part considérable de familles mixtes, les différentes formes possibles de relations de parenté (de sang ou par alliance) avec un haut niveau de variation suivant les localités.

This article presents an experience of intercultural contact between the Chuvash, who speak a Turkic language, and Finno-Ugric peoples (Mari, Udmurt and Mordvinian) in different regions of the Ural-Volga area. It is based on the author's fieldwork. Its starting point is the understanding of ethnicity as a form of social organisation of cultural difference, an understanding that defines the features of interethnic interaction, its forms, mechanisms and consequences. In the relations between the Chuvash and Finno-Ugrians, there are some general features. From the point of view of language, there is an increasing bilingualism that is becoming multilingualism. As far as ethnic culture is concerned, there is syncretism in the culture of the religious rituals: the traditional sets integrate elements from the neighbour's culture, which over time becomes their own; we can also notice a high level of intercultural competence and the ability to overcome religious distance and organise common rituals. At the same time, there is a tendency towards the religious, cultural and linguistic isolation of ethnic groups, visible in the territorial structure of mixed villages, in the organisation of cultural space, in the ethnic preferences in contacts and in stereotypes in the perception of neighbours. The social sphere is characterised by mutual socio-cultural adaptation at different levels, by the high percentage of mixed marriages, by the existence of many mixed families and the different forms of kinship relations (by blood or marriage) with a high level of local variation.

В СТАТЬЕ РАССМОТРЕН ОПЫТ МЕЖКУЛЬТУРНОГО ВЗАИМОДЕЙСТВИЯ ТЮРКОЯЗЫЧНЫХ ЧУВАШЕЙ С ФИННО-УГОРСКИМИ НАРОДАМИ (МАРИЙЦАМИ, УДМУРТАМИ, МОРДВОЙ) В РАЗЛИЧНЫХ РАЙОНАХ УРАЛО-ПОВОЛЖЬЯ. ИССЛЕДОВАНИЕ ПРОВЕДЕНО НА ОСНОВЕ ПОЛЕВЫХ МАТЕРИАЛОВ АВТОРА. АНАЛИЗ МАТЕРИАЛА ОСНОВАН НА ПОНИМАНИИ ЭТНИЧНОСТИ КАК ФОРМЫ СОЦИАЛЬНОЙ ОРГАНИЗАЦИИ КУЛЬТУРНЫХ РАЗЛИЧИЙ, ОПРЕДЕЛЯЮЩИХ ХАРАКТЕР МЕЖЭТНИЧЕСКОГО ВЗАИМОДЕЙСТВИЯ, ЕГО ФОРМЫ, МЕХАНИЗМЫ И РЕЗУЛЬТАТЫ. В ОТНОШЕНИЯХ ЧУВАШЕЙ С ФИННО-УГОРСКИМИ НАРОДАМИ НАБЛЮДАЮТСЯ СЛЕДУЮЩИЕ ЗАКОНОМЕРНОСТИ. В ЯЗЫКОВОЙ 
СФЕРЕ СУЩЕСТВУЕТ БИЛИНГВИЗМ С ТЕНДЕНЦИЕЙ И ПРИМЕРАМИ ПРЕВРАЩЕНИЯ ЕГО В ПОЛИЯЗЫЧИЕ. В СФЕРЕ ЭТНИЧЕСКОЙ КУЛЬТУРЫ - СИНКРЕТИЗМ В РЕЛИГИОЗНО-ОБРЯДОВОЙ КУЛЬТУРЕ, ПРИЗНАКАМИ КОТОРОГО ВЫСТУПАЮТ ВЗАИМОПРОНИКНОВЕНИЕ В ТРАДИЦИОННЫЕ КОМПЛЕКСЫ ГРУПП ЭЛЕМЕНТОВ КУЛЬТУРЫ СОСЕДА - КОТОРЫЕ НАЧИНАЮТ ВОСПРИНИМАТЬСЯ СО ВРЕМЕНЕМ В КАЧЕСТВЕ «СВОИХ»-, ВЫСОКИЙ УРОВЕНЬ МЕЖКУЛЬТУРНОЙ КОМПЕТЕНТНОСТИ, ПРЕОДОЛЕНИЕ РЕЛИГИОЗНОЙ ОТЧУЖДЕННОСТИ ГРУПП И ТРАДИЦИЯ СОВМЕСТНОГО ПРОВЕДЕНИЯ ОБРЯДОВ ЭТНИЧЕСКИМИ И ЭТНОКОНФЕССИОНАЛЬНЫМИ ГРУППАМИ. В ТО ЖЕ ВРЕМЯ НАБЛЮДАЕТСЯ ТЕНДЕНЦИЯ РЕЛИГИОЗНОГО И КУЛЬТУРНО-ЯЗЫКОВОГО ОБОСОБЛЕНИЯ ЭТНИЧЕСКИХ ГРУПП, ПРОЯВЛЯЮЩАЯСЯ В ТЕРРИТОРИАЛЬНО-ПОСЕЛЕНЧЕСКОЙ СТРУКТУРЕ СМЕШАННЫХ СЕЛЕНИЙ, ОРГАНИЗАЦИИ КУЛЬТУРНОГО ПРОСТРАНСТВА, ЭТНИЧЕСКИЕ ПРЕДПОЧТЕНИЯ В МЕЖЭТНИЧЕСКИХ КОНТАТКАХ, В СТЕРЕОТИПАХ ВОСПРИЯТИЯ СОСЕДЕЙ. ДЛЯ СОЦИАЛЬНОЙ СФЕРЫ ХАРАКТЕРНА ВЗАИМНАЯ СОЦИОКУЛЬТУРНАЯ АДАПТАЦИЯ ЭТНИЧЕСКИХ И КОНФЕССИОНАЛЬНЫХ ГРУПП НА РАЗНЫХ УРОВНЯХ СОЦИАЛЬНОГО ВЗАИМОДЕЙСТВИЯ, ВЫСОКИЙ УРОВЕНЬ МЕЖЭТНИЧЕСКОЙ БРАЧНОСТИ, НАЛИЧИЕ ЗНАЧИТЕЛЬНОЙ ДОЛИ ЭТНИЧЕСКИ СМЕШАННЫХ СЕМЕЙ, РАЗЛИЧНЫХ ВАРИАНТОВ РОДСТВЕННЫХ И СВОЙСТВЕННЫХ ОТНОШЕНИЙ С ВЫСОКОЙ СТЕПЕНЬЮ ВАРИАТИВНОСТИ ПО СЕЛЕНИЯМ.

\section{INDEX}

nomsmotscles Bachkirs, Maris, Maris des collines, Maris des plaines, Mordves, Oudmourtes, Russes, Tatars, Tchouvaches

Keywords : assimilation, bilingualism, ethnic identity, ethnicity, intercultural contacts, mixed marriages, religions, ritual culture, syncretism

Thèmes : ethnologie, oralités

Mots-clés : assimilation, bilinguisme, contacts interculturels, culture rituelle, ethnicité, identité ethnique, mariages mixtes, religions, syncrétisme

Index chronologique : XXIe siècle (début)

Index géographique : Aurgazy, Bachkortostan, Bahtigildino, Bakaj, Batyrevo, Bavli, Bazlyk, Bižbuljak, Buguruslan, Buzuluk, Čekmaguš, Čuvašskaja Kulatka, Čuvašskaja Rešetka, Čuvaš-tary, Dijaševo, Eremkino, Ermekeevo, Gornomari, Homuter', Hvalynsk, Ibresi, Il'terjakovo, Jumaševo, Kalamantaevo, Kalmantaj, Kargino, Karmaskaly Malye Karmaly, Mari El, Mičurinsk, Mordovskij Šmalak, Muhino, Naumkino, Nikulino, Nižnjaja Tuarma, Orenbourg, Pisarevo, Podlesnaja Andreevka, Poreckoe, Potapovo-Tumbarla, Sakty, Salejkino, Samara, Šaran, Saratov, Semenovka, Semurša, Šentala, Staraja Lebežajka, Staryj Afon'kino, Sukkulovo, Tatarstan, Tchouvachie, Timjaševo, Trehbaltaevo, Trojary, Udmurtskie Tašly, Uljanovsk, Verhnjaja Tuarma, Zirgan, Živajkino, Zvenigorsk

disciplines bachkir, mari, mordve, tchouvache, oudmourte, russe, tatar motsclesru АССИМИЛЯЦИЯ, БИЛИНГВИЗ, МЕЖКУЛЬТУРНЫЕ КОНТАКТЫ, ОБРЯДЧЕСКАЯ КУЛЬТУРА, РЕЛИГИЯ, СИНКРЕТИЗМ, СМЕШЕННЫЕ БРАКИ, ЭТНИЧЕСТВО, ЭТНИЧЕСКАЯ ИДЕНТИЧНОСТЬ

motscleset assimileerimine, kakskeelsus, etniline identiteet, etnilisus, kultuurivahelised kontaktid, religioon, rituaalne kultuur, segaabielud, sünkretism 